LA-UR-01-1414

Approved for public release; distribution is unlimited.

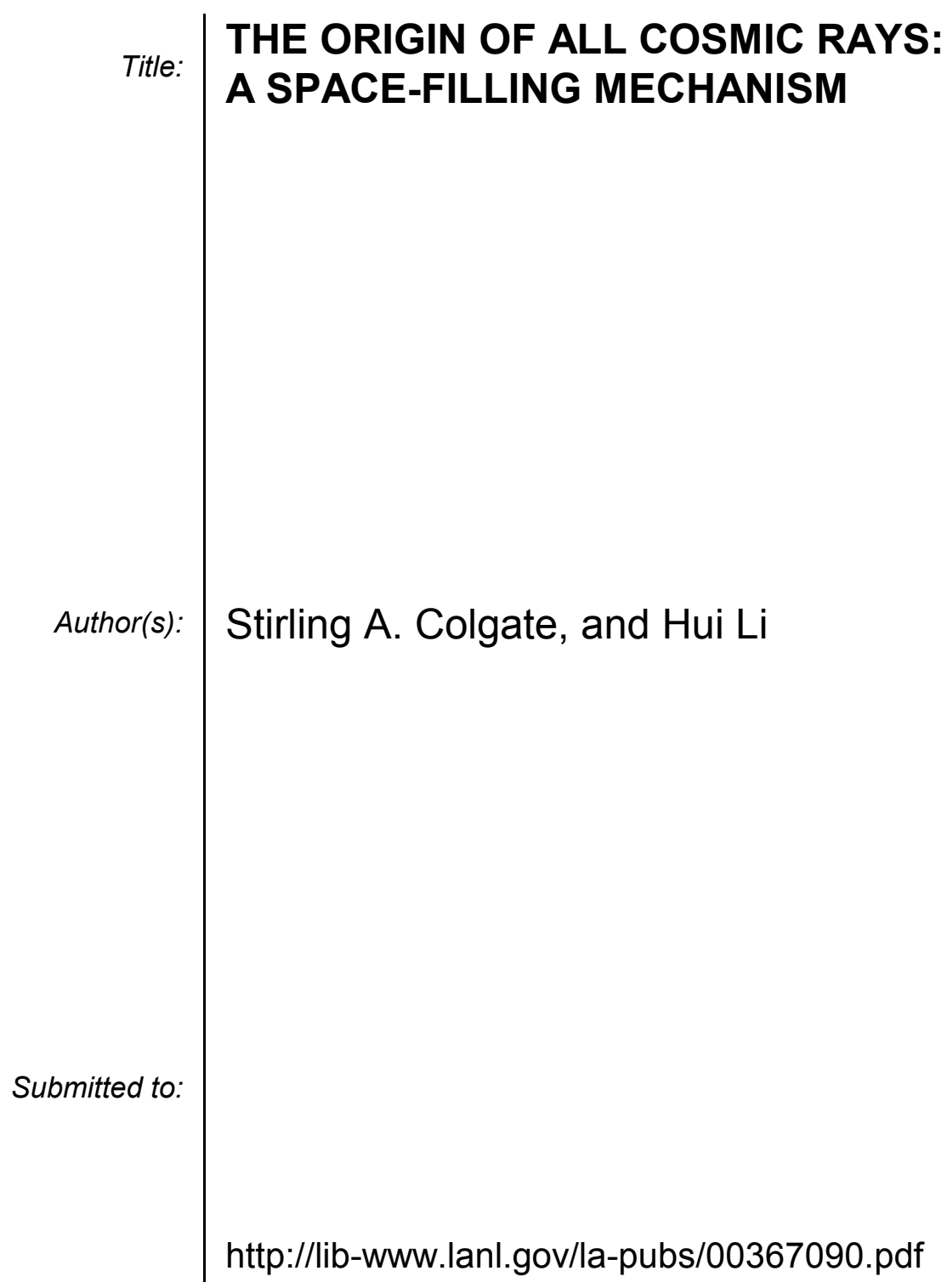

Los Alamos National Laboratory, an affirmative action/equal opportunity employer, is operated by the University of California for the U.S. Department of Energy under contract W-7405-ENG-36. By acceptance of this article, the publisher recognizes that the U.S. Government retains a nonexclusive, royaltyfree license to publish or reproduce the published form of this contribution, or to allow others to do so, for U.S. Government purposes. Los Alamos National Laboratory requests that the publisher identify this article as work performed under the auspices of the U.S. Department of Energy. Los Alamos National Laboratory strongly supports academic freedom and a researcher's right to publish; as an institution, however, the Laboratory does not endorse the viewpoint of a publication or guarantee its technical correctness. 


\section{THE ORIGIN OF ALL COSMIC RAYS: A SPACE-FILLING MECHANISM}

Stirling A. Colgate, and Hui Li*

We suggest that all cosmic rays are accelerated by a uniformly distributed, space-filling mechanism. based upon the reconnection of force-free magnetic fields. (1) The current astronomical description of feasible point sources either galactic or extra-galactic (e.g., supernovae or AGN) leads to an inconsistently large CR anisotropy compared to observations. (2) A space-filling mechanism implies a universal acceleration mechanism since there is only one feasible form of space-filling free energy, namely a force-free magnetic field. (3) A single mechanism, compared to several, implies nearly the same energy spectrum, galactic as well as extra-galactic, in which case the extra-galactic total energy requirement demands that nearly all the possible or feasible free energy of the universe be accessed to create this particle energy spectrum down to its maximum at a Gev. (4) This largest accessible free energy is the energy released in galactic black hole formation, $10^{\wedge} 62$ ergs per galaxy, which is converted to forcefree magnetic field by the galactic black hole accretion disk dynamo. (6) Nearly $100 \%$ efficiency of conversion of BH energy to magnetic is just sufficient to account for an extra galactic spectrum similar to the observed galactic one. and with sufficiently frequent reacceleration ( 100 times during $\mathrm{t}$ (Hubble)) in order to hide the 
UHCRs forming the GZK cut-off. feature in the spectrum by loss to the voids, the only place where they can be lost and not return to be observed. (7) Therefore the acceleration mechanism must also be nearly $100 \%$ efficient in the conversion of force-free field energy to particle energy. (8) The only possible fashion by which to access magnetic force-free energy is by the electrical power from $J$ (parallel) Idot $\mathrm{E}$ (parallel). (The parallel component is by definition of forcefree.) (9) Therefore the sum of all CRs must be most of the $\mathrm{J}$ (parallel) of all force-free flux in the universe. Then their individual energy depends upon their individual dwell time within the $\mathrm{E}$ (parallel) fields. (10) In this view the cosmic rays are the result of a statistical dwell time of a particle within the E(parallel) associated with the reconnection of the force-free fields of the universe. (11) Since the extragalactic CR energy is so much greater than the galactic CR energy, Isim Itimes $10^{\wedge}(5$ to 6$)$, we tend to focus on the extra galactic power. However, within the galaxy the twisting of all advected magnetic flux during the accretion leading to star formation and the similar twisting by magnetized, rotating neutron stars leads to more than sufficient accessible force-free magnetic energy to power and accelerate the CRs within our galaxy.

\begin{abstract}
:
There is a need for one mechanism to accelerate cosmic rays universally over the full energy spectrum, isotropically, and space filling. The current view is a theory based upon a series of mechanisms, patched to fit various spectral regions with a mechanism for the origin of the UHCRs still in doubt. We suggest that the reconnection of force-free magnetic fields produced by the twisting of all imbedded magnetic flux by the vorticity motion of all accretion or condensations both within the Galaxy as well as the metagalaxy is the
\end{abstract}


universal mechanism. This leads to the acceleration of all cosmic rays with both total energy and individual energies up to the highest observed of $3 \times 10^{20} \mathrm{ev}$ and predicting an upper limit of $10^{23} \mathrm{ev}$. There are three primary, and we believe compelling reasons for adopting this different view of the origin of CRs. (1) The energy source is space filling and isotropic, thereby avoiding any anisotropy's due to single sources, e.g., supernovae remnants and AGN. (2) The galactic and particularly the extragalactic energy source is sufficient to supply the full energy of a universal galactic and extragalactic spectrum of $10^{60}$ to $10^{61} \mathrm{ergs}$ sufficient to avoid the GZK cut-off. (3) Efficient Eparallel acceleration from reconnection of force-free fields is well observed in the laboratory whereas collisionless shock acceleration still eludes laboratory confirmation.

\section{Introduction}

The widely accepted mechanism of collisionless shock acceleration is most frequently substantiated by the prediction of a power-law spectrum not much different from that observed. Instead we observe that any non-thermal acceleration mechanism that results in a fractional loss in number of particles for a given fractional gain in energy results in a power -law spectrum . Whatever mechanism of acceleration that results in the minimum fractional loss of particles per fractional gain in particle energy results in that particular spectral index that is a minimum index value. The number of particles at higher energy then will exceed those of a steeper (more negative index) spectrum. This will then will be selectively chosen as the preferred mechanism for producing the higher energy particles.

*T-6, MSB-288, LANL, Los Alamos, NM87545 colgate@lanl.gov

Reconnection is the dissipation of the parallel currents that maintain the force-free fields, resulting in a parallel electric field and hence parallel acceleration. Magnetic energy is stored primarily in the tension of force-free fields. It is released or converted only by the dissipation, Eparallel $\mathrm{x}$ Jparallel, of the currents that maintain the magnetic stressed (force-free) state. The sources of such twisted forcefree field energy and flux are the many mass condensations, which 
always occur with finite angular momentum, e.g., accretion disks or rotating stars. The large winding number during the dissipation of this angular momentum assures that all imbedded flux will necessarily undergo continuous winding until either the angular momentum is dissipated or reconnection takes place. This assures that at least several orders of magnitude of twist i.e., a winding number of at least several hundred or greater will take place.

The transition between this picture of transient, multiple, highly twisted, flux-tubes within our galaxy (and between galaxies) and the observed state of relatively ordered flux must occur by reconnection. The acceleration of cosmic rays in this picture is then a sum of both individual particles that are accelerated in single coherent regions of high winding number and random diffusion in energy space of particles that stochastically traverse many such coherent regions of Eparallel acceleration (and deceleration.) The advantage of reconnection acceleration is that it is well grounded in laboratory measurements e.g. tokamak interruptions, whereas shock acceleration is still an unfounded speculation.

\section{Why do we need an alternate Theory of the Origin of Cosmic Rays?}

1) The observed isotropy of CRs requires a space-filling mechanism both for galactic CRs as well as for the UHCRs of extra galactic origin. If supernova shocks are the sources within our galaxy, then the diffusion mean-free path for galactic CRs after acceleration must be very long, >1 kpc, and yet not escape the galaxy. The spacing between the few near-by supernovae in the CR life-time is large enough to lead to an observed anisotropy. In order that the spatial fluctuations and therefore anisotropy due to a small number of contributing supernova be small enough. and furthermore that the surrounding "leaky box" be extraordinarily isotropic. The same argument applies to the UHCRs if they are accelerated locally within AGN. 
2) The plasma physics of the required scattering mechanism necessary to produce shock acceleration is unlikely because of individual particle damping within the large non-linear amplitude waves of the turbulence necessary for the scattering itself. The average number of scatterings per particle required for shock acceleration of one e-fold in relativistic energy is approximately $\left(\mathrm{v}_{\text {shock}} / \mathrm{c}\right)^{2} \sim 10^{5}$ to $10^{6}$ scatterings. These scatterings must occur from strong, self-excited, hydromagnetic waves with a loss per scattering of less than $10^{-5}$ to -6 per doubling in energy. Such a small loss or high reversibility is very unlikely unless proven in the laboratory. The hydromagnetic turbulence must be "strong" in order that a particle be scattered within a few Larmor orbits, $\sim 10$, in dimension, which are then the mean free path of each scattering event.

3) The evidence for acceleration due to quasi-parallel heliosphere shocks from satellite measurements near the earth is weak, in some cases with small or no evidence for the expected strong hydromagnetic turbulence, and where acceleration is demonstrated only after a few shock traversals by the accelerated particles. Thus no more than $10 \mathrm{kT}$ acceleration has been observed whereas $10^{20} \mathrm{kT}$ must be achieved.

4) Laboratory experiments necessary to demonstrate shock acceleration have not yet succeeded and space observations have failed to observe the necessary turbulence of the collisionless shock.

5) The argument that a unique power-law spectra resulting from shock acceleration is not compelling.

6) The above argument of number of scatterings per shock crossing and a non-linear wave amplitude of B/delta B 10, implies a shock thickness of

$\mathrm{n}$ RLarmor $=10$ (c/vshock) RLarmor $=1 \mathrm{kpc}$ at $1015 \mathrm{ev}$, the "knee" of the spectrum, and therefore a thickness significantly greater than the thickness of the galaxy. 


\section{Power-law Spectrum}

The accepted theory of cosmic ray acceleration is shock wave acceleration in the ISM driven by supernova (Axford, Leer, and Skadron, 1977; Bell, 1978; Blanford and Ostriker, 1988). "This acceptance has been largely based upon the good agreement between the "universal" power-law spectrum predicted by shock acceleration i.e., the power-law index becomes:

$$
\mathrm{s}=(\mathrm{d} \ln \mathrm{N}) /(\mathrm{d} \ln \mathrm{E}) \sim-(2+\mathrm{e})
$$

depending only on the Mach number and the observed or inferred particle spectra." (see Blanford and Eichler, 1987 for a review, and many papers by P. Biermann for a more accurate comparison.) This belief that a nearly correct power-law spectral index alone is unique is instead, a less restrictive condition than commonly believed. Any accelerator for which a fractional gain in energy, (d ln E), by a few particles is accompanied by a fractional loss, $-(\mathrm{d} \ln \mathrm{N})$, in number of the remainder will give a power-law:

$$
\mathrm{dN} / \mathrm{N}=-\mathrm{s}(\mathrm{dE} / \mathrm{E}) \text {. }
$$

The fractional loss for a fractional gain in energy is what would be expected for a rigidity dependent loss mechanism where the probability of a relativistic particle being scattered out of an acceleration region is inversely proportional to its energy or rigidity.

For values of $s<\sim 2$, i.e. a smaller fractional loss, the integral energy becomes asymptotically large, $\int_{0}^{\mathrm{E}} \mathrm{NdE} \approx \mathrm{E}^{s-2}$ and at some energy will truncate or limit the acceleration mechanism, destroying the confinement and hence the accelerating mechanism itself. Hence, it is not likely that at any one time we should see many such accelerators occurring naturally in the Galaxy. On the other hand accelerators with $\mathrm{s} \gg 2$ will produce a steep spectrum that, relative to another less steep one, i.e. $\mathrm{s}$ closer to 2 , will be lost relative to the less steep mechanisms 
above some critical energy. Hence it is likely that the spectrum of any observed mechanism should be close to $\mathrm{s}=2+\mathrm{e}$.

\section{Isotropy}

Any mechanism for accelerating cosmic rays that depends primarily upon supernova will have a problem producing the necessary isotropy presently observed (Richardson and Osborn, 1974). From a Tev to 10 to 100 times greater, where shower arrays are most sensitive to anisotropy, this anisotropy is $10^{-3}$ or less. Within the lifetime, $t$, of cosmic rays of $10^{7}$ years and a diffusion coefficient of $\mathrm{D}$, there is a characteristic distance, $\mathrm{d}$, within which particles can diffuse to the observer. The anisotropy of the diffusive flux will be of the order of the maximum fractional differences of the source distribution of

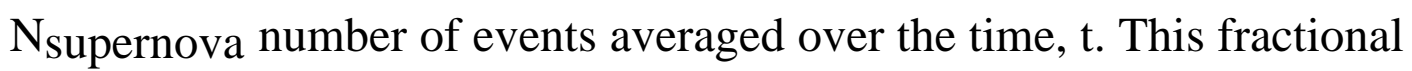
difference is of order $\left(\mathrm{N}_{\mathrm{sn}}\right)^{1 / 2}$. If all the supernova within the galaxy at a typical rate of $1 / 30$ years in the life time of $10^{7}$ years, or $3 \times 10^{5}$ supernova were all equally contributing to the local flux, then the anisotropy would be of the order $\left(3 \times 10^{5}\right)^{-1 / 2}=1.8 \times 10^{-3}$ a value somewhat larger than observed. The diffusion distance, $d$, must then be significantly greater than the dimension of the galaxy, $30 \mathrm{kpc}$. Under these circumstances the diffusion coefficient must be greater than $3 \times 10^{31} \mathrm{~cm}^{2} / \mathrm{s}$ or a mean free path for scattering greater than $1 \mathrm{kpc}$. This is $3 \times 10^{4}$ greater than what is assumed for the necessary scattering for the diffusive shock mechanism where the mean free path is frequently assumed to be roughly 10 Larmor radii at $10 \mathrm{Tev}$. If this difference in scattering length indeed occurs due to a decrease in hydromagnetic turbulence after the passage of the shock, then one is faced with the further difficulty that the cosmic rays would escape from the galaxy unless the whole galaxy were surrounded by a near perfect "leaky box" with an anisotropic imperfection of diffusive loss of less than $5 \times 10^{-4}$. This is hard to imagine in view of current galactic dynamo theory where a dipole field surrounds the Galaxy yet at the same time inside the box cosmic rays diffuse relatively easily. If a smaller diffusion coefficient, D, is imagined, then in a flat Galaxy, 
$\mathrm{N}_{\mathrm{Sn}}$ is proportional to $\mathrm{D}$ and the probable anisotropy due to fluctuations becomes large, proportional to $\mathrm{D}^{-1 / 2}$. Hence an unlikely circumstance must exist of first a very small diffusion coefficient, then a very large one, and finally a near perfectly symmetric surrounding leaky diffusive box.

\section{Particle Scattering Losses}

It is well recognized that in diffusive shock acceleration a particle must traverse the shock many times, c/vshock $~ 300$ in order to double in energy. However, in order to return to the shock this number of times by random scattering, the mean number of scattering events becomes the square of this number of crossings, or $10^{5}$ scatterings. One recognizes that the accelerated particles are likely to be the exceptional ones that cross the shock the requisite number of times with fewer scatterings, but nevertheless the fractional loss per scattering must be very small for the average particle, which is the particle "lost" from acceleration by diffusion to convection at each energy. Hence, recognizing the many orders of magnitude of acceleration required, in order not to modify the power-law exponent below the good agreement with theory, the fractional irreversible loss per scattering must be less than $\sim 10^{-5}$ to -6 . This is an extraordinarily small loss in the presence of what must be strong turbulence in order to have the short scattering lengths required ( 10 Larmor orbits) to reach the highest energies (Lagage and Caesarsky). One suspects the excitation of at least one mode of non-linear damping of the hydromagnetic waves at the level of

$10^{-5}$ to -6 . Such non-linear damping induces heating and hence damping of those particles furthest ahead of the shock that excite the waves in the first place. The particles furthest ahead are those of greatest rigidity and therefore most sensitive to modifying the spectrum.

\section{The Reconnection Theory of Acceleration}

The specific angular momentum of matter in the galaxy rotating at an $\mathrm{w}=10^{-8}$ per year and a local radius corresponding to roughly one 
solar mass, $(\sim 3 \mathrm{pc})$ is $3 \times 10^{22} \mathrm{~cm}^{2} / \mathrm{s}$. This is some $2 \times 10^{4}$ larger than the Keplearian orbit at a solar radius. This factor of angular momentum must be exchanged with the Galaxy as a whole in order to form a star, and still more angular momentum must be removed in order to collapse to a neutron star. The most likely mechanism for the transport of this angular momentum is by a turbulent, a-viscosity, the Rossby vortex mechanism, (Li et al. 2001) in the disk-like accretion of a star through the T-tauri stage. (If the torque is transmitted by magnetic stress, this makes the following effects even larger.). Similarly the magnetic flux threading the original matter at the density of the ISM is also orders of magnitude (300) larger than the flux that would allow compression to a solar radius. Hence magnetic flux must also be expelled from the condensing matter as well. Therefore this flux, in order to escape the matter, must undergo reconnection during the process of condensation. However the subsequent winding or twisting of this residual flux requires a far larger reconnection process since the winding produces a far larger total flux than the original.

The picture at every stage of condensation is that of a helical, twisted flux tube that extends some 50 to 100 turns beyond the source. The number of turns is interpreted observationally from the topology of the "jets" or collimated radio sources which are presumed to be just these helical flux tubes from accretion onto the black holes of AGN. They become illuminated in the radio by the accelerated electrons due to the reconnection of the force-free flux of the twisted flux tube. The same picture applies to the bi-polar flows observed in star formation. The relation between the number of turns and the angle of the "jet" is that the minimum energy force-free field of the helix is close to a 45 degrees where the $\mathrm{f}$ and $\mathrm{z}$ components of the field are equal. Hence the number of turns, $n$, will be of the order (1/jet angle) $x \ln \left(L_{\max } / R_{\min }\right)$, or several hundred.

One possible location to accelerate the UHECRs, E> $1018 \mathrm{ev}$, is in the force-free field helix next to the black hole of nearly every galaxy. Here it is assumed that the reconnection dissipates the total current associated with the $\mathrm{B}_{\mathrm{f}}$ component of the field. If this current is 
interrupted as a sequence of multiple reconnection events, but phased along a local flux surface at $\mathrm{c}$ by the accelerated particles themselves, the total potential becomes of the order:

$$
\mathrm{V}=\mathrm{n} \mathrm{B}_{\max } \mathrm{R}_{\min } 300(2 \mathrm{pln} \mathrm{n})=2 \times 10^{23} \mathrm{ev} .
$$

where $\mathrm{B}_{\max }=10^{4}$ gauss, and $\mathrm{R}_{\min }=1$ au.

Individual near-by $(100 \mathrm{Mpc})$ AGN sources should then be seen in the UHECR distributions, contrary to observations.

\section{Extra Galactic Acceleration}

Our current view (Colgate and Li 2000, 2001) is that this forcefree helical field fills the intergalactic space with magnetic flux, $\sim 10^{-6}$ $\mathrm{G}$ per galaxy that decays by reconnection over a Hubble time. The total energy of $\sim 3 \times 10^{61}$ ergs per galaxy spacing volume $\left(\sim 10^{74} \mathrm{~cm}^{3}\right)$ is close to the limiting energy that can be supplied by the black hole and is observed as the minimum energy _necessary to supply the synchrotron radiation of many radio lobes. This energy is also only slightly greater than the energy of the extra-galactic spectrum itself, assuming the UHECRs are extrapolated back to a Gev with the typical galactic index of -2.7. If one uses the slightly flatter slope of -2.5 implied by the spectrum E> $1018 \mathrm{ev}$, then there is enough energy in the intergalactic fields on this model to re accelerate the CRs $\sim 100$ times during a Hubble time. These particles should then be confined primarily to the galaxy walls with a loss to the voids in $1 / 100$ of the Hubble time. Because of this rapid loss to the voids of CRs accelerated in the walls, the remaining young population of UHCRs should then not be subject to the GZK cut off in the spectrum . This lost to the voids occurs by random walk in the coherent helical fields produced by each galaxy. The same upper energy limit as above, close to the black hole should apply in the IGM because the magnetic flux is presumed to be the same.

\section{Summary}


Accretion onto galactic black holes, neutron stars, cataclysmic variables, and T-tauri stars will all lead to tearing mode reconnection associated with twisted fields by all condensations. The energy of these condensations is far more than required to maintain the cosmic rays of our Galaxy.

Laboratory experiments can be performed to simulate both magneto hydrodynamics as well as the tearing mode reconnection and the associated Eparallel acceleration of the "run-away" particles. The spheromak experiments are a step in this direction. Interruptions in tokomaks are already laboratory proof of this acceleration. We need to perform more laboratory plasma experiments to observe reconnection in the near vacuum limit with the resulting parallel electric fields. Without laboratory experiments, as for example shock acceleration, we are still uncertain about the origin of cosmic rays. 


\section{REFE3RENCES}

Axford,W.I., Leer,E., and Skadron, G., 1977, ICRC.. 15th ICRC, 11, 132.

Bell, A.R., 1978, MNRAS, 182,147.

Blanford, R.D. and Ostriker, J.P., 1988, ApJ, 221, L29.

Blanford, R.D. and Eichler, D., 1987, Physics Reports, 154,1,1987.

Colgate, S.A. and Li, Hui, 1999, Astrophys. Space Sci. , 264. 357

Colgate, S.A. and Li, Hui, 2000, "The Magnetic Fields of the Universe and Their Origin" IAU Symp 195, ASP Conf. Series 334, eds. P.C.H. Martens and S. Tsurta 1999

Colgate, S.A. and Li, H. \& Pariev,V., 2001,"The Origin of the Magnetic Fields of the Universe:The Plasma Astrophysics of the Free Energy of the Universe" Physics of Plasmas, accepted

Jokipii, J.R., Morfill, G., 1987, Astrophys. J. 312, 170

Lagage, P.O., Cesarsky, C.J., 1983, Astron. \& Astroph. 118, 223 